\title{
European Renal Best Practice Guideline on kidney donor and recipient evaluation and perioperative care
}

\author{
Daniel Abramowicz ${ }^{1}$, Pierre Cochat ${ }^{2,3}$, Frans H.J. Claas ${ }^{4}$, Uwe Heemann ${ }^{5}$, Julio Pascual ${ }^{6}$, C. Dudley ${ }^{7}$, \\ Paul Harden ${ }^{8}$, Marivonne Hourmant $^{9}$, Umberto Maggiore ${ }^{10}$, Maurizio Salvadori ${ }^{11}$, Goce Spasovski ${ }^{12}$, \\ Jean-Paul Squifflet $^{13}$, Jürg Steiger ${ }^{14}$, Armando Torres ${ }^{15,16,17}$, Ondrej Viklicky ${ }^{18}$, Martin Zeier ${ }^{19}$, \\ Raymond Vanholder ${ }^{20}$, Wim Van Biesen ${ }^{21}$ and Evi Nagler ${ }^{22}$
}

${ }^{1}$ Department of Nephrology, Antwerp University Hospital, Antwerp, Belgium, ${ }^{2}$ Hopital Femme Mere Enfant, Universite de Lyon, Lyon, France, ${ }^{3}$ Service de Nephrologie Pediatrique, Centre de Reference des Maladies Renales Rares, Inserm U820, Faculte de medecine RTH Laennec, Universite de Lyon, Lyon, France, ${ }^{4}$ Department of Immunohaematology and Blood Transfusion, Leiden University Medical Center, Leiden, the Netherlands, ${ }^{5}$ Department of Nephrology, Klinikum rechts der Isar, Munich, Germany, ${ }^{6}$ Department of Nephrology, Hospital del Mar Barcelona, Barcelona, Spain, ${ }^{7}$ Richard Bright Renal Unit, Southmead Hospital, Bristol, UK, ${ }^{8}$ Department of Nephrology, Churchill Hospital, Oxford University, Oxford, UK, ${ }^{9}$ Service de Nephrologie, CHU Nantes, Nantes Cedex, France, ${ }^{10}$ UO Nefrologia, Trapianti Rene-Pancreas, Parma, Italy, ${ }^{11}$ Renal Unit, Azienda Ospedaliero-Universitaria Careggi, Florence, Italy, ${ }^{12}$ Medical Faculty, University Department of Nephrology, Skopje, Republic of Macedonia, ${ }^{13}$ Department of Abdominal Surgery and Transplantation, CHU Liege, Liège, Belgium, ${ }^{14}$ Transplantation Immunology and Nephrology, University Hospital Basel, Basel, Switzerland, ${ }^{15}$ Center for Biomedical Research of the Canary Islands (CIBICAN), University of La Laguna, La Laguna, Spain, ${ }^{16}$ Nephrology Service, University Hospital of Canary Islands, La Laguna, Spain, ${ }^{17}$ Nefrología, Instituto Reina Sofía de Investigación, Madrid, Spain, ${ }^{18}$ Department of Nephrology, Institute for Clinical and Experimental Medicine, Prague, Czech Republic, ${ }^{19}$ Department of Nephrology, University of Heidelberg, Heidelberg, Germany, ${ }^{20}$ Department of Nephrology, University Hospital Ghent, Ghent, Belgium, ${ }^{21}$ Dienst Nefrologie, UZ Gent, Gent, Belgium and ${ }^{22}$ Renal Division, University Hospital Ghent, Ghent, Belgium

Correspondence and offprint requests to: Dr. Daniel Abramowicz; E-mail: daniel.abramowicz@uza.be

\section{ABSTRACT}

The European Best Practice Guideline group (EBPG) issued guidelines on the evaluation and selection of kidney donor and kidney transplant candidates, as well as post-transplant recipient care, in the year 2000 and 2002. The new European Renal Best Practice board decided in 2009 that these guidelines needed updating. In order to avoid duplication of efforts with kidney disease improving global outcomes, which published in 2009 clinical practice guidelines on the post-transplant care of kidney transplant recipients, we did not address these issues in the present guidelines.

The guideline was developed following a rigorous methodological approach: (i) identification of clinical questions, (ii) prioritization of questions, (iii) systematic literature review and critical appraisal of available evidence and (iv) formulation of recommendations and grading according to Grades of Recommendation Assessment, Development, and Evaluation (GRADE). The strength of each recommendation is rated 1 or 2 , with 1 being a 'We recommend' statement, and 2 being a 'We suggest' statement. In addition, each statement is assigned an overall grade for the quality of evidence: A (high), B (moderate), C (low) or D (very low). The guideline makes recommendations for the evaluation of the kidney transplant candidate as well as the potential deceased and living donor, the immunological work-up of kidney donors and recipients and perioperative recipient care.

All together, the work group issued 112 statements. There were $51(45 \%)$ recommendations graded ' 1 ', $18(16 \%)$ were graded ' 2 ' and $43(38 \%)$ statements were not graded. There were 
$0(0 \%)$ recommendations graded ' $1 \mathrm{~A}$ ', $15(13 \%)$ were ' $1 \mathrm{~B}$ ', 19 (17\%) ' $1 \mathrm{C}$ ' and 17 (15\%) '1D'. None (0\%) were graded ' $2 \mathrm{~A}$ ', 1 $(0.9 \%)$ was ' $2 B$ ', 8 (7\%) were ' $2 \mathrm{C}$ ' and $9(8 \%)$ ' $2 \mathrm{D}$ '. Limitations of the evidence, especially the lack of definitive clinical outcome trials, are discussed and suggestions are provided for future research.

We present here the complete recommendations about the evaluation of the kidney transplant candidate as well as the potential deceased and living donor, the immunological work-up of kidney donors and recipients and the perioperative recipient care. We hope that this document will help caregivers to improve the quality of care they deliver to patients. The full version with methods, rationale and references is published in Nephrol Dial Transplant (2013) 28: i1-i71; doi: 10.1093/ndt/ gft218 and can be downloaded freely from http://www. oxfordjournals.org/our_journals/ndt/era_edta.html.

Keywords: donor evaluation, ERBP, guideline, kidney transplantation, perioperative care, recipient evaluation

\section{INTRODUCTION}

Caring for kidney transplant recipients (KTRs) requires specialized knowledge in areas as varied as nephrology, immunology, pharmacology, endocrinology, infectious disease and cardiology. In this context of increasing complexity coupled with an exponential growth in the medical literature, clinical practice guidelines (CPGs) aim at helping clinicians and other caregivers to deliver evidence-based medicine and thereby, to improve patient outcomes. Furthermore, guidelines also help to expose gaps in our knowledge and thereby suggest areas where additional research is needed. This guideline was developed following a rigorous methodological approach: (i) identification and selection of a representative work group, consisting of experts in transplantation (nephrologists, surgeons and immunologists) and guideline methodologists; (ii) identification of clinical questions; (iii) prioritization of questions; (iv) systematic literature review and critical appraisal of available evidence; (v) formulation of recommendations and grading according to GRADE; (vi) comparison to existing guidelines, when available and (vii) suggestions for future research. The GRADE system allows provision of guidance even if the evidence base is weak, but makes the quality of the available evidence transparent and explicit. The strength of each recommendation is rated 1 or 2 , with 1 being a 'We recommend' statement implying that most patients should receive the course of action, and 2 being a 'We suggest' statement implying that different choices will be appropriate for different patients with the suggested course of action being a reasonable choice. In addition, each statement is assigned an overall grade for the quality of evidence: A (high), B (moderate), C (low) or $\mathrm{D}$ (very low). Although there are reasons other than quality of evidence to make a grade 1 or 2 recommendation, in general, there is a correlation between the quality of overall evidence and the strength of the recommendation. Even if the evidence is weak, clinicians still need to make clinical decisions in their daily practice, and they often ask 'what do the experts do in this setting'? Therefore, the European Renal Best Practice (ERBP) board opted to give guidance, even if evidence was weak or non-existent, which unfortunately is often the case in nephrology. The draft guidelines were submitted for review to selected European experts, all European Renal Association European Dialysis and Transplantation Association (ERAEDTA) members and reviewers selected by the European Society of Organ Transplantation and The Transplantation Society. Where appropriate, changes based on these comments were made in the final document. We felt this is an important step in the development of guidelines, as it fuelled the base of expertise that enhanced the overall quality of the guideline. We owe a special debt of gratitude to all those who took time out of their busy schedules to share their comments with us. They have been instrumental in improving the final guidelines.

We hope that this document will help caregivers to improve the quality of care they deliver to patients.

Daniel Abramowicz, Transplantation work group Co-chair

Wim Van Biesen, ERBP advisory board Chairman

Pierre Cochat, Transplantation work group Co-chair

Raymond Vanholder, President of ERA-EDTA

\section{CORE RECOMMENDATIONS}

Chapter 1. Evaluation of the kidney transplant candidate 1.1 Should we actively screen for the presence of malignancy in kidney transplant candidates? Is presence or history of malignancy a contra-indication to kidney transplantation?

1.1.1. We recommend screening kidney transplant candidates for cancer according to the recommendations that apply to the general population. (Ungraded Statement)

1.1.2. We suggest screening kidney transplant candidates for the presence of kidney cancer by ultrasound. (Ungraded Statement)

1.1.3. We suggest screening for the presence of urothelial cancer by urinary cytology and cystoscopy in kidney transplant candidates with an underlying kidney disease associated with an increased risk of this type of cancer. (Ungraded Statement)

1.1.4. We recommend screening $\mathrm{HCV}$ and $\mathrm{HBV}$-infected kidney transplant candidates for the presence of hepatocellular carcinoma according to the EASL-EORTC Clinical Practice Guideline on the management of hepatocellular carcinoma. (Ungraded Statement)

1.1.5. We suggest that patients with current or previous cancer should be discussed with an oncologist and considered on a case-by-case basis. The following factors should be considered when determining the appropriate time that waitlisting should be delayed: (a) the potential for progression or recurrence of the cancer according to its type, staging and grade; (b) the age of the patient; (c) the existence of co-morbidities, in order to define the appropriate period of time that wait-listing should be delayed. (Ungraded Statement) 
1.2 Under which conditions can HIV-infected patients be enrolled on the waiting list?

1.2.1. We recommend that HIV per se is not a contra-indication for kidney transplantation. (1C)

1.2.2. We recommend wait-listing HIV patients only if

(1) they are compliant with treatment, particularly HAART therapy.

(2) their CD $4+\mathrm{T}$-cell counts are $>200 / \mu \mathrm{L}$ and have been stable during the previous 3 months.

(3) HIV RNA was undetectable during the previous 3 months.

(4) no opportunistic infections occurred during the previous 6 months.

(5) they show no signs compatible with progressive multifocal leukoencephalopathy, chronic intestinal cryptosporidiosis or lymphoma. (1C)

1.2.3. We suggest that the most appropriate anti-retroviral therapy should be discussed before transplantation with the infectious diseases team in order to anticipate potential drug interactions after transplantation. (Ungraded Statement)

1.3 Is there a role for immunization against herpes varicella zoster prior to kidney transplantation?

1.3.1. We recommend immunization against varicella-zoster virus in all paediatric and adult patients negative for antivaricella-zoster antibodies, preferably when they are still waitlisted. (1D)

1.4 Should haemolytic uraemic syndrome (HUS) as underlying cause of end-stage kidney disease preclude wait-listing for transplantation and does it influence graft and patient survival post-transplantation?

1.4.1. We recommend that typical, proven shiga-toxin E. coliassociated haemolytic uraemic syndrome (HUS) is not contra-indication to transplantation from either deceased or living donors. (1B)

1.4.2. We suggest considering kidney transplantation as an acceptable option (i) in kidney transplant candidates with atypical HUS and a proven membrane cofactor protein (MCP) mutation, and (ii) in those displaying anti-complement factor $\mathrm{H}(\mathrm{CFH})$ auto-antibodies. (Ungraded Statement)

1.4.3 We suggest that kidney transplantation in patients with atypical HUS should only be undertaken in centres with experience in managing this condition and where appropriate therapeutic interventions are available. (Ungraded Statement)

1.4.4. We do not recommend living donation from a genetically related donor in patients who are suspected to have atypical HUS as their underlying kidney disease unless the responsible mutation has been conclusively excluded in the donor. (1D)

1.4.5. We recommend evaluating the potential of living donation from a genetically unrelated donor to a recipient with atypical HUS on a case-by-case basis. It should only be considered after appropriate counselling of the recipient and donor on the risk of disease recurrence in the transplanted graft. (Ungraded Statement)

1.5 Should focal segmental glomerulosclerosis (FSGS) as underlying cause of end-stage kidney disease preclude waitlisting for transplantation and does it influence graft and patient survival post-transplantation?

1.5.1. We recommend that primary focal segmental glomerulosclerosis per se is not a contra-indication to kidney transplantation from either a living or a deceased donor. (1D)

1.5.2. We recommend informing the recipient and in living donation, the potential donor, about the risk of recurrence of focal segmental glomerulosclerosis in the graft. (Ungraded Statement)

1.5.3. We recommend that when a first graft has been lost from recurrent focal segmental glomerulosclerosis, a second graft from either a deceased or a living donor should only be transplanted after an individual risk/benefit assessment and careful counselling of the recipient and potential donor in the case of living donation. (Ungraded Statement)

1.5.4. We suggest using an updated management protocol in cases of recurrent focal segmental glomerulosclerosis. (Ungraded Statement)

1.5.5. We suggest that children with steroid-resistant nephrotic syndrome undergo appropriate genotyping before wait-listing them for kidney transplantation. (Ungraded Statement)

1.6 Does pre-transplant alcohol and drug abuse in patients influence patient or graft survival?

1.6.1. We recommend that women who drink $>40 \mathrm{~g}$ and men who drink $>60 \mathrm{~g}$ of alcohol per day stop or reduce their alcohol consumption to below these levels. (1D)

1.6.2. These patients can be waitlisted, but careful surveillance of the reduction of alcohol consumption should be exerted. (Ungraded Statement)

1.6.3. We recommend that patients with alcohol 'dependence' should not be waitlisted. (Ungraded Statement)

1.6.4. Strategies to stop alcohol consumption should be offered, according to the WHO Clinical Practice Guideline. (Ungraded Statement)

1.6.5. We recommend that patients with an on-going addiction to 'hard drugs' resulting in non-adherence should not be waitlisted for transplantation. (1D)

1.7 Does pre-transplant tobacco smoking in patients influence patient or graft survival?

1.7.1. We recommend that patients stop smoking before transplantation. (1B) 
1.7.2. Smoking cessation programmes should be offered. (Ungraded Statement)

1.8 Should obesity preclude wait-listing for kidney transplantation and is there a difference in outcomes post-transplantation between those with and without obesity?

1.8.1. We recommend that patients with a body mass index $>30 \mathrm{~kg} / \mathrm{m}^{2}$ reduce weight before transplantation. (Ungraded Statement)

1.9 Should kidney transplantation be delayed in patients presenting with uncontrolled secondary hyperparathyroidism? Does uncontrolled secondary hyperparathyroidism in the immediate pre-transplant period have an impact on transplant outcomes?

1.9.1. We recommend not refusing a cadaveric graft only because of uncontrolled hyperparathyroidism in the recipient. (1D)

1.9.2. However, for patients on the waiting list, efforts should be made to comply with existing chronic kidney diseasemetabolic bone disease guidelines, including parathyroidectomy, when indicated. (Ungraded Statement)

1.10 How should screening for potential cardiovascular disease in the potential recipient be done in a cost-effective way?

1.10.1. We recommend that basic clinical data, physical examination, resting ECG and chest-X ray are a sufficient standard work-up in asymptomatic low-risk kidney transplant candidates. (1C)

1.10.2. We recommend performing a standard exercise tolerance test and cardiac ultrasound in asymptomatic high-risk patients (older age, diabetes, history of cardiovascular disease). In patients with a true negative test, further cardiac screening is not indicated. (1C)

1.10.3. We recommend performing further cardiac investigation for occult coronary artery disease with non-invasive stress imaging (myocardial perfusion or dobutamine stress echocardiography) in kidney transplant candidates with high risk and a positive or inconclusive exercise tolerance test. (1C)

1.10.4. We recommend performing coronary angiography in renal transplant candidates with a positive test for cardiac ischaemia. Further management should be according to the current cardiovascular guidelines (Figure 1). (1D)

1.11 When and for which indications should native nephrectomy be performed in kidney transplant candidates awaiting kidney transplantation?

1.11.1. We recommend native nephrectomy before transplantation (unilateral or bilateral) in patients with autosomal polycystic kidney disease (ADPKD) when there are severe, recurrent symptomatic complications (bleeding, infection, stones). (1C)
1.11.2. We suggest unilateral nephrectomy of asymptomatic ADPKD kidneys when space for the transplant kidney is insufficient. (2C)

1.11.3. We do not recommend routine native nephrectomy, unless in cases of recurrent upper urinary tract infections or when the underlying kidney disease predisposes to enhanced cancer risk in the urogenital tract. (Ungraded Statement)

Chapter 2. Immunologic work-up of kidney donors and recipients

2.1. How should HLA typing be performed in renal transplant candidates and donors?

2.1.1. We suggest that at least one typing is performed by molecular human leucocyte antigen (HLA) typing of patients and donors to avoid mistakes in the classification of the HLA antigens. (2D)

2.1.2. We suggest that HLA typing is performed in duplicate, preferentially on separate samples obtained at different occasions to avoid logistical errors. (Ungraded Statement)

2.1.3. In case of sensitized patients, we recommend additional serological typing of the donor cells to be used for crossmatches in order to check the proper expression of the HLA antigens on the target cells. (1D)

2.1.4. For highly sensitized patients with allele-specific antibodies we suggest consideration of high resolution molecular typing in both recipients and donors. (2D)

2.2. In a renal transplant recipient, how should HLA matching be used to optimize outcome?

2.2.1. We suggest matching for human leucocyte antigen (HLA)-A, -B and -DR whenever possible. (2C)

2.2.2. We recommend balancing the effects of HLA matching with other parameters that affect patient and graft outcomes when deciding the acceptance of a potential graft. (1D)

2.2.3. We recommend giving preference to an HLA identical donor and recipient combination. (1B)

2.2.4. We suggest giving more weight to HLA-DR matching than to HLA A and B matching. (2C)

2.2.5. We recommend giving more weight to HLA matching in younger patients, in order to avoid broad HLA sensitization which might impair re-transplantation. (Ungraded Statement)

2.3. In renal transplant candidates, what HLA antigens and non-HLA antigens should be defined in addition to HLA A, $B$ and DR?

2.3.1. We recommend performing human leucocyte antigen (HLA) DQ, HLA DP and HLA C typing of the donor only when the intended recipient has HLA antibodies against those antigens. (1D)

2.3.1. We do not recommend routine typing for major histocompatibility complex class I related chain-A (MICA) and other non-HLA antigens in either the recipient or donor. 


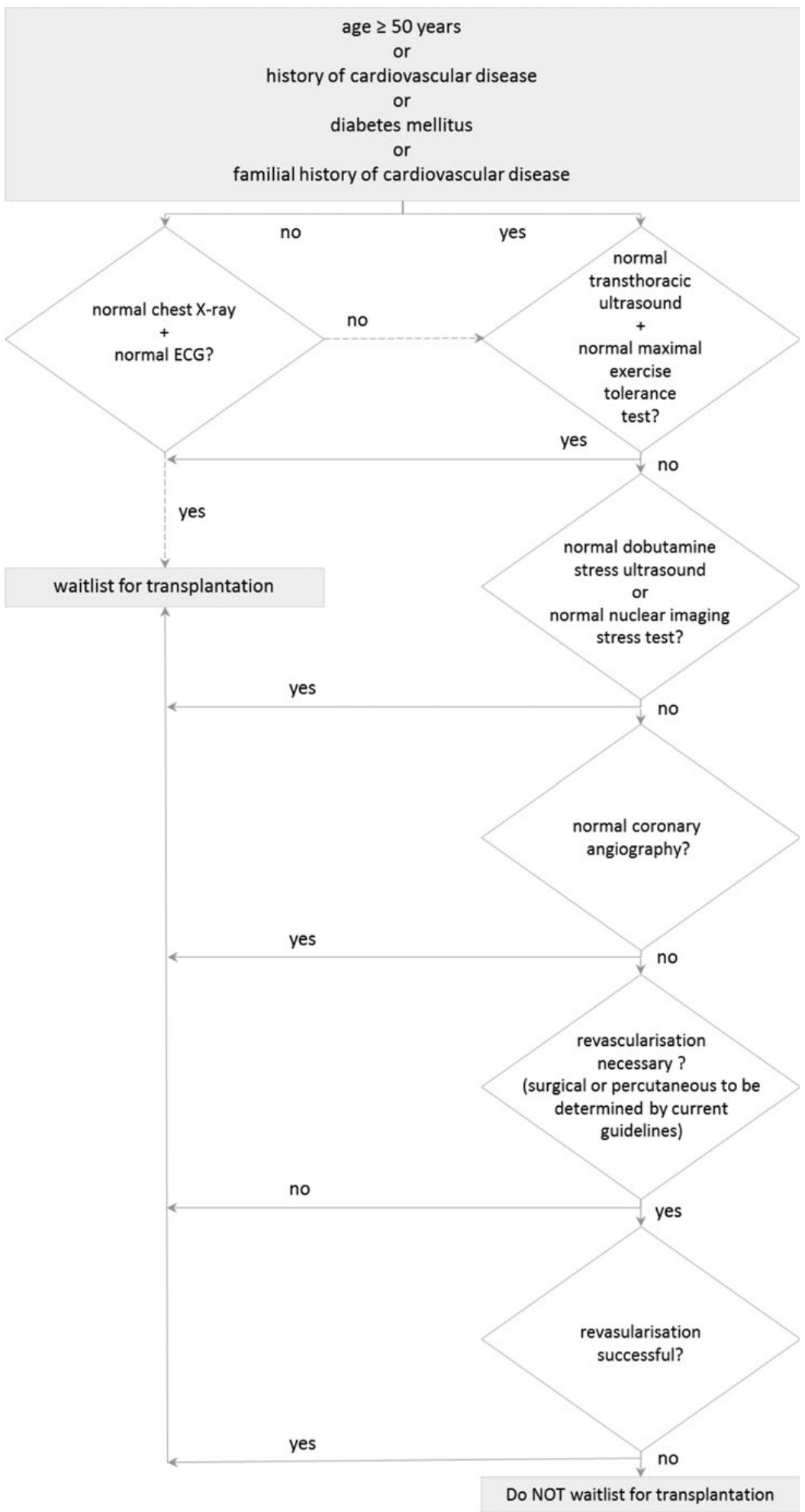

F I G U R E 1. Decision tree pre-transplant cardiovascular screening.

2.4. In HLA-sensitized kidney transplant candidates what measures should be attempted to improve the probability of a successful transplantation?

2.4.1. We recommend establishing programmes to select a donor towards whom the recipient does not produce antibodies. (1C)
2.4.2. In recipients from cadaveric kidney donors, this aim can be achieved by an acceptable mismatch programme. (1C)

2.4.3. In living donation this goal can be achieved by paired exchange. (Ungraded Statement)

2.4.4. We recommend transplanting patients with donor-specific antibodies only if these abovementioned measures cannot be accomplished and after successful intervention. (1D) 
2.5. In renal transplant candidates, should a failed allograft that is still in place be removed or left in place?

2.5.1. Evidence comparing patients with a failed transplant with versus without nephrectomy is insufficient and conflictive, hampering a meaningful general recommendation on whether or not nephrectomy of failed grafts should be recommended. (Ungraded Statement)

2.5.2. We suggest that in the following conditions an explantation of the failed kidney graft be considered: clinical rejection, chronic systemic inflammation without other obvious cause or recurrent (systemic) infections. (Ungraded Statement)

2.5.3. We suggest to continue low-level immunosuppression and to avoid nephrectomy of a failed graft when residual graft urinary output is $>500 \mathrm{~mL} /$ day and there are no signs of inflammation. (Ungraded Statement)

2.6. In renal transplant candidates, what technique of crossmatch should be used to optimize outcomes?

2.6.1. We recommend performing a complement-dependent cytotoxic (CDC) crossmatch in HLA-sensitized patients to prevent hyperacute rejection. (1B)

2.6.2. We suggest that in HLA antibody negative patients with negative regular quarterly screening samples a crossmatch can be omitted, unless a potential HLA sensitizing event has occurred since last screening. (2B)

2.6.3. We do not recommend performing a Luminex or endothelial cell crossmatch because their additional value needs further evaluation. (1D)

2.6.4. We recommend that a positive CDC crossmatch should only be accepted as truly positive when donor-specific antibodies are known to be present. (1B)

2.7. In renal transplant candidates planned to undergo living donor transplantation but for whom the available donor is $\mathrm{ABO}$ incompatible, what measures can be taken to improve outcome after transplantation?

2.7.1. We recommend both inhibition of antibody production and $\mathrm{ABO}$ antibody removal before transplantation applied together in one and the same validated protocol. (1C)

2.7.2. We recommend transplantation of an $\mathrm{ABO}$ incompatible kidney only if the $\mathrm{ABO}$ antibody titre after intervention is lower than $1: 8$. (1C)

2.7.3. We suggest considering paired exchange when available. (Ungraded Statement)

2.8. In previously transplanted patients, what is the effect of repeated mismatches for HLA antigens on outcome, as compared to avoiding repeated HLA mismatches?

2.8.1. We recommend that repeated human leucocyte antigen (HLA) mismatches are not considered a contra-indication for transplantation in the absence of antibodies against those repeated mismatches. (Ungraded Statement)

2.8.2. We suggest that the presence of antibodies against the repeated mismatch detectable by other techniques than the complement-dependent cytotoxic (CDC) technique be considered as a risk factor rather than a contra-indication. (Ungraded Statement)

Chapter 3. Evaluation, selection and preparation of deceased and living kidney donors

3.1. When is dual kidney transplantation preferred over single kidney transplantation?

3.1.1. We recommend that before the kidneys of a cadaveric donor are discarded because they are deemed unsuitable for single transplantation, transplantation of both kidneys into one recipient (dual kidney transplantation) is considered as an option. (1C)

3.1.2. We suggest that in cadaveric donors where there is uncertainty about the quality of the kidneys, the decision to either discard the kidneys, or use them as a dual or a single transplant, is based on a combination of the clinical evaluation and history of the recipient and donor, and when available, a standardized assessment of a pre-transplant donor biopsy. (2D)

3.1.3. We recommend that before a kidney from a paediatric donor is discarded due to low donor age it is deemed unsuitable for single transplantation in an adult recipient, en bloc transplantation is considered. (1B)

3.1.4. We suggest that the option of using kidneys for en bloc transplantation is always considered for donors weighing less than $10 \mathrm{~kg}$. (1D)

3.2. Which perfusion solution is best suited for kidney preservation in recipients of living donation? Which perfusion solution is best suited for kidney preservation in recipients of deceased kidney donation?

3.2.1. There is insufficient evidence to favour a particular preservation solution for kidneys that carry a low risk of delayed graft function. (Ungraded Statement)

3.2.2. We recommend not using Eurocollins as a preservation solution for kidneys that carry a high risk of delayed graft function (long projected cold ischaemia time, extended criteria donors). (1B)

\subsection{Is machine perfusion superior to standard perfusion?}

3.3.1. There are conflicting data regarding the generalizability of the benefit of machine perfusion over static cold storage. Until further evidence emerges, no firm recommendation for the use of machine perfusion in preference to cold storage can be made. (Ungraded Statement) 
3.4. Is there a critical cold ischaemia time beyond which a donated organ should be discarded?

3.4.1. We suggest that cold ischaemia time be kept as short as possible. (2D)

3.4.2. We recommend keeping cold ischaemia time below 24 hours when transplanting kidneys from donors after brain death. (1B)

3.4.3. We recommend keeping cold ischaemia time to less than 12 hours when using kidneys from donors after cardiac death. (1D)

3.4.4. We recommend that the decision to use donor kidneys with a cold ischaemia time of more than 36 hours be made on a case per case basis. (1D)

3.5. On which criteria should we select living kidney donors to optimize the risk/benefit ratio of their donation?

\section{General remarks}

3.5.1. We recommend encouraging living kidney donors to exercise on a regular basis and when relevant, to lose weight and stop smoking. (1C)

3.5.2. We recommend that the individual risk of donation should be carefully discussed with the donor, taking into account the situation of both donor and recipient. Ideally, this should be done using standardized checklists to ensure all items are discussed. (Ungraded Statement)

3.5.3. We suggest that the donor be evaluated by an independent physician who is not part of the transplant team and is not involved in the daily care of the recipient, and when possible, by a psychologist. (Ungraded Statement)

3.5.4. We recommend that the process of donation is stopped should any doubt on donor safety arise, especially in younger donors, or when the benefit for the recipient is limited. (Ungraded Statement)

3.5.5. We recommend that the simultaneous presence of more than one risk factor (hypertension, obesity, proteinuria, impaired glucose tolerance, haematuria) precludes donation. (Ungraded Statement)

\section{Hypertension}

3.5.6. We recommend considering potential donors with a blood pressure $<140 / 90 \mathrm{mmHg}$ on at least three occasions without antihypertensive medication, as normotensive. (1C)

3.5.7. We suggest measuring ambulatory blood pressure in potential donors who have office hypertension (blood pressure $\geq 140 / 90 \mathrm{mmHg}$ ) or who are taking pharmacological treatment for hypertension. (2C)

3.5.8. We suggest well-controlled primary hypertension, as assessed by ambulatory blood pressure $<130 / 85 \mathrm{mmHg}$, under treatment with maximum two anti-hypertensive drugs (diuretics included) is not considered a contra-indication to living kidney donation. (2C)
3.5.9. We recommend declining hypertensive donors with evidence of target organ damage such as left ventricular hypertrophy, hypertensive retinopathy and microalbuminuria. (1C)

3.5.10. We suggest that these potential donors be re-evaluated for disappearance of this target organ damage after appropriate treatment. (2D)

\section{Obesity}

3.5.11. We suggest a body mass index above $35 \mathrm{~kg} / \mathrm{m}^{2}$ is a contra-indication to donation. (2C)

3.5.12. We recommend counselling obese and overweight donors for weight loss before and after donation. (Ungraded statement)

\section{Impaired glucose tolerance}

3.5.13. We recommend diabetes mellitus is a contra-indication to donation, other than in exceptional circumstances. (1D)

3.5.14. We suggest impaired glucose tolerance is not an absolute contra-indication to donation. (2C)

\section{Proteinuria}

3.5.15. We recommend to quantify urinary protein excretion in all potential living donors. (1C)

3.5.16. We recommend overt proteinuria is a contra-indication for living donation [24-hour total protein $>300 \mathrm{mg}$ or spot urinary albumin to creatinine $(\mathrm{mg} / \mathrm{g})$ ratio $>300(>30 \mathrm{mg} /$ $\mathrm{mmoL})] .(1 \mathrm{C})$

3.5.17. We recommend further evaluating potential living donors with persistent (more than three measurements with 3 months interval) proteinuria $<300 \mathrm{mg} / 24 \mathrm{~h}$ by quantification of albuminuria to assess their risk of living donation. (Ungraded statement)

3.5.18. We suggest considering persistent (more than three measurements with 3 months interval) moderate albuminuria (30-300 mg/24 h) a high risk for donation. (Ungraded statement)

\section{Haematuria}

3.5.19. We recommend considering persistent haematuria of glomerular origin as a contra-indication to living donation, because it may indicate renal disease in the donor. (1B)

3.5.20. However, we acknowledge thin basement membrane disease might be an exception. (Ungraded statement)

\section{Old age}

3.5.21. We recommend that old age in itself is not a contra-indication to donation. (1B) 
3.6. What lower level of kidney function precludes living donation?

3.6.1. We recommend that all potential living kidney donors should have their glomerular filtration rate (GFR) assessed. (1C)

3.6.2. We recommend that in cases where more exact knowledge on GFR is needed or where is doubt regarding the accuracy of GFR from estimation methods, a direct measurement of GFR should be undertaken by exogenous clearance methods. (Ungraded Statement)

3.6.3. We recommend that all potential donors should have a predicted GFR that is projected to remain above a satisfactory level after donation within the life-time of the donor as indicated in the graph below. (Ungraded Statement)

3.7. What are the risks of pregnancy in a woman with a single kidney after living kidney donation?

3.7.1. We recommend informing women of childbearing age that as they are a selected from a very healthy subpopulation, donation increases their individual risk from below that of the general population, to that of the general population. (1B)

3.8. What is the best surgical approach for living donor nephrectomy for the donor? What is the best surgical approach for living donor nephrectomy for the recipient?

3.8.1. For living donor nephrectomy we suggest either a minimally invasive or laparoscopic approach rather than a flank subcostal retroperitoneal one. The choice between minimal invasive and laparoscopic procedure should be based on the local expertise. (2C)

\section{Chapter 4. Perioperative care of the kidney transplant} recipient

4.1. What are the indications for an additional haemodialysis session in the recipient immediately before the transplantation procedure?

4.1.1. We recommend not routinely performing a haemodialysis session immediately before the actual transplantation procedure unless there are specific clinical indications. (1C)

4.1.2. When additional haemodialysis is performed immediately before the transplantation procedure, we recommend not using ultrafiltration unless there is evidence of fluid overload. (1C)

4.2. Does the use of central venous pressure measurement as a guidance tool for fluid management in kidney transplant recipients improve the outcome after transplantation?

4.2.1. We suggest that central venous pressure be measured and corrected in the early post-operative period to prevent hypovolemia and delayed graft function. (2D)
4.3. In kidney transplant recipients during the perioperative period, does the use of intravenous solutions other than $0.9 \%$ sodium chloride improve patient and/or graft outcome?

4.3.1. There is no evidence to prefer one type of solution (crystalloids versus colloids, normal saline versus Ringer) for intravenous volume management of the recipient during kidney transplant surgery.

4.3.2. In view of the available data in the literature, and in line with the ERBP position on prevention of AKI, we suggest to be cautious with the use of starches in the kidney transplant recipient during the perioperative period, although specific data in this setting are lacking. (Ungraded Statement)

4.3.3. We recommend monitoring for metabolic acidosis when normal saline is used as the only intravenous fluid in the perioperative and post-operative period. (1B)

4.4. Does the use of dopaminergic agents (dopamine and its alternatives) improve early post-operative graft function?

4.4.1. We do not recommend the use of 'renal doses' of dopaminergic agents in the early post-operative period, since it does not improve graft function or survival. (1B)

4.5. Should we use prophylactic antithrombotic agents during the perioperative period?

4.5.1. We do not recommend routinely using low-molecularweight heparin, unfractionated heparin or aspirin before transplantation to prevent graft thrombosis. (1B)

4.6. In renal transplant recipients, what are the effects of using a JJ stent at the time of operation on renal outcomes?

4.6.1. We recommend prophylactic JJ stent placement as a routine surgical practice in adult kidney transplantation. (1B)

4.6.2. We suggest that if a JJ stent is in place, cotrimoxazole is given as antibiotic prophylaxis. (2D)

4.6.3. We suggest removing the JJ stent within 4-6 weeks. (Ungraded Statement)

4.7. What is the optimal post-operative time for removal of the indwelling bladder catheter in kidney transplant recipients?

4.7.1. We suggest removing the urinary bladder catheter as soon as possible after transplantation, balancing the risk of urinary leak against that of urinary tract infection. (2D)

4.7.2. We recommend monitoring adverse event rates (urinary tract infection, urinary leakage) in each centre, to inform the decision over when to remove the indwelling bladder catheter. (1D)

Received for publication: 9.5.2014; Accepted in revised form: 12.5.2014 